\author{
Heike Behrens
}

\title{
Grammatik und Lexikon im Spracherwerb: Konstruktionsprozesse ${ }^{1}$
}

Putting together novel expressions is something that speakers do, not grammars. It is a problemsolving activity that demands a constructive effort and occurs when linguistic convention is put to use in specific circumstances.

(Langacker 1987, S. 65)

\begin{abstract}
Der Konstruktionsbegriff hielt seinen Einzug in die Spracherwerbsforschung durch gebrauchsbasierte Lerntheorien, nach denen sprachliche Strukturen als Form-Funktionseinheiten aus dem Input abgeleitet werden, Sprache somit ein emergentes System ist (Tomasello 1998a und b; Behrens 2009a und b). Die Abstraktionseinheit für das Kind ist dabei die Äußerung in ihrer situativen Gebundenheit und ihrer Diskursfunktion, mithin die Konstruktion. Die Konstruktion wird gefasst als schematische Einheit mit mehr oder weniger offenen Slots: Teile der Konstruktion können lexikalisch fixiert oder aber produktiv und durch andere Ausdrücke ersetzbar sein. Der Kontrast zum Valenzbegriff bzw. dem der Argumentstruktur in seiner formaleren Definition liegt darin, dass die lexikalischen Eigenschaften der Wörter die Syntax nicht projizieren, sondern dass sowohl die Eigenschaften der Lemmas als auch die der Morphosyntax aus ihrem Vorkommen in konkreten Sätzen abgeleitet werden.

Empirisch konzentriert sich die Forschung auf die Ermittlung der Generalisierungsprozesse und auf deren Basis im Input, dem Sprachangebot. Erwerbsrelevant ist insbesondere der Input in seinen usualisierten Mustern in typischen Interaktionssituationen. Hier wird vor allem der Grad der Produktivität kindlicher Äußerungen analysiert. Bislang weniger untersucht, aber zunehmend im Fokus sind die Generalisierungsprozesse selbst und damit die generative Kraft des Konstruktionsbegriffs. Sobald Aspekte einer Konstruktion abstrahiert worden (= produktiv) sind, sollten sie auf neue Situationen übertragen werden können, und gilt es zu ermitteln, welche formalen, funktionalen und distributionellen Faktoren die Abstraktion sprachlichen Wissens fördern.

In dem Paradigma der gebrauchsbasierten Konstruktionsgrammatik wird die modulare Trennung zwischen Wörtern und Regeln aufgehoben. Somit kann innerhalb eines einheitlichen theoretischen Rahmens sowohl der Erwerb regelhafter als auch der stärker idiosynkratischer Strukturen erklärt werden.
\end{abstract}

Die Arbeit an diesem Aufsatz wurde unterstützt durch eine Senior Fellowship am Freiburg Institute for Advanced Studies (FRIAS). 


\section{Lexikon und Grammatik im Spracherwerb}

Die Rolle des Lexikons im Syntaxerwerb aus konstruktivistischer Perspektive möchte ich anhand von zwei unterschiedlichen Themenfeldern beleuchten: Erstens dem Erwerb der Argumentstruktur, der sich direkt auf den Tagungstitel „Sprachliches Wissen zwischen Lexikon und Grammatik“ bezieht. Hier untersucht man den Zusammenhang zwischen den semantisch bedingten Subkategorisierungseigenschaften des Verbs und der syntaktischen Realisierung der Argumente, insbesondere den Einfluss dieser Beziehung auf den Spracherwerbsprozess. Zweitens dem Erwerb der Satzklammer im Deutschen mit den unterschiedlichen Positionen für finite und nichtfinite Verben je nach Satztyp. Dieses Phänomen scheint zunächst unabhängig vom Lexikon zu sein, weil prinzipiell alle Verben dieser Wortstellungssystematik unterliegen, auch wenn es statistische Unterschiede dahingehend geben mag, wie oft die einzelnen Verbformen für einzelne Verben realisiert werden. Ich möchte zeigen, wie konstruktivistische, gebrauchsbasierte Ansätze zum Spracherwerb den Erwerb eher semantik- und eher morphosyntax-gesteuerter Phänomene in einem homogenen Framework erklären können.

\section{Der Erwerb der Argumentstruktur}

Das Erkenntnisziel der Spracherwerbsforschung ist, zu erklären, wie Kinder Sprache in ihrer Totalität erwerben. Um dieses Ziel zu erreichen, braucht man eine (Arbeits)definition dessen, was Sprache ist (Durch welche Kategorien ist sie instantiiert? Wie ist sie mental repräsentiert?), Hypothesen zu den Lernprozessen (Mittels welcher kognitiven Prozesse lernt das Kind Sprachstruktur und Sprachfunktion?) und schließlich ein methodisches Instrumentarium, mit dem sich die Hypothesen, die sich aus den Grundannahmen ableiten können, testen lassen.

Mit diesem Forschungsprogramm unterscheidet sich die Spracherwerbsforschung von anderen Gebieten der Linguistik, insofern als sie nicht nur die Stadien der Sprachentwicklung adäquat beschreiben, sondern auch erklären können muss, wie die postulierten Strukturen gelernt werden können. Spracherwerbsmodelle sind also notwendigerweise auch kognitive Modelle.

Innerhalb nativistischer Ansätze zum Spracherwerb steht die Arbeitsteilung zwischen Wörtern und Grammatik im Vordergrund. In „words and rules"-Modellen (z.B. Pinker 1989) werden Wörter nach Regeln der Grammatik zu Sätzen zusammengesetzt. Diese Sichtweise ist charakteristisch für die Sicht der generativen Grammatik auf den Spracherwerb, sowohl in Hinblick auf den Erwerb von grammatischen Regeln, die als unabhängig von semantischen oder funktionalen Faktoren konzeptualisiert werden, als auch 
in einer stärkeren lexikalistischen Ausrichtung in Studien zum Erwerb der Argumentstruktur. Sofern substantielle (repräsentationelle) Universalien angenommen werden, muss hier das Linking-Problem gelöst werden. LinkingProzesse müssen stattfinden, um die angeborenen Strukturen mit den Elementen der Zielsprache zu verknüpfen. Lernen durch Linking ist dann eindeutig, wenn alle Verben durch ein solches Regelsystem erfasst werden können. Sofern ein Teil des Lexikons bzw. der Argumentstruktur nicht durch solche Regeln vorhersagbar ist und idiosynkratisch im Lexikon gespeichert werden müsste, wirkt sich das auf den Lernprozess aus, denn nun muss erklärt werden, wie diese idiosynkratischen Eigenschaften gelernt werden (hier wird in der Regel Gebrauchsbasiertheit angenommen), und wie die sprachliche Repräsentation aussieht, die als universal und angeboren vorausgesetzt wird. D.h., man endet bei hybriden Modellen, die einerseits inputbasiert, andererseits aber gebrauchs- und sprachunabhängig sind (vgl. Hohenberger/Pelzer-Karpf 2009). Diese Hybridität löst das Erwerbs- und Linking-Problem jedoch nicht, sondern verschärft es noch, denn es erfordert zwei Lernprozesse (Linking und Generalisierung). Zudem muss erklärt werden, auf welcher Basis das Kind wissen könnte, wann es den einen oder anderen Prozess zu aktivieren hat, wann es auf die Generalisierung über den Sprachgebrauch verzichten kann, weil dieser Aspekt der Sprache gebrauchsunabhängig erworben wird. Generell gibt es keine Evidenz dafür, dass durch die Universalgrammatik (UG) kontrollierte Aspekte früher oder schneller gelernt werden als nicht-UG-gebundene; die Tatsache dass Spracherwerb zügig und zielgerichtet voranschreitet, ist keine Evidenz für Angeborenheit (Behrens 2009b).

In gebrauchsbasierten Ansätzen werden Form-Funktionsbeziehungen aus dem Sprachangebot abstrahiert. Dies impliziert, dass resultierende Kategorien sprachspezifisch und gebrauchsspezifisch sein sollten. Operationalisiert wird dieser Ansatz z.B. im Paradigma der „Preferred Argument Structure“ (PAS), das einzelsprachlich und sprachvergleichend die diskurspragmatischen Faktoren, die zur Realisierung oder Ellipsis von Argumenten führen, analysiert (vgl. Allen/Skarabela/Hughes 2008 sowie die Artikel in Bowerman/Brown (Hg.) 2008). In der Sprachtypologie ist umstritten, ob es tatsächlich universale Muster der Argumentstrukturen gibt, nach denen eine bestimmte Bedeutung generell mit bestimmten Subkategorisierungsmustern einhergeht (vgl. Bowerman/Brown 2008). Gebrauchspräferenzen in der Argumentrealisierung wirken sich auch auf den Erwerb der Wortarten aus: In der Debatte, ob Nomen prinzipiell vor Verben erworben werden, weil sie referenziell sind, Verben jedoch relationale Informationen ausdrücken (Gentner 1978), erweist sich, dass in Sprachen mit starker Subjekt- und/oder Objekt-Ellipsis Verben automatisch frequenter und salienter auftreten. Zudem kann der Verbwortschatz auf lexikalisch sehr spezifischen Verben bestehen wie im Tzeltal mit einem sehr reichen Vokabular an Verben 
des Essens, des Bewegens usf., oder aus semantisch eher leeren und eher wenigen Verben wie in einigen australischen Aboriginal-Sprachen. Die semantische Spezifik sowie die perzeptuelle Prominenz durch Frequenz und Salienz erleichtern den Einstieg in die Kategorie Verb (Brown 1998).

Im Folgenden werden die Prozesse des Erwerbs der Argumentstruktur, wie sie sich aus nativistischer ,top down“- bzw. gebrauchsbasierter „,bottom up"-Perspektive ergeben, kontrastiert.

\subsection{Universales Linking als Einstieg in den Syntaxerwerb}

In der Spracherwerbsforschung der achtziger und neunziger Jahre galten die Subkategorisierungseigenschaften von Verben als verbindende Kraft zwischen formalen und funktionalen Aspekten des Sprachsystems und damit als eine Schnittstelle im Erwerb der Syntax und Semantik, die in den so genannten Bootstrapping-Ansätzen konzeptualisiert wurde. Bahnbrechend wirkten die Überlegungen Steven Pinkers (Pinker 1987, 1989, 1994; Gropen et al. 1991), der postulierte, dass die Semantik Kindern zum Einstieg in die Syntax verhilft. Innerhalb deduktiver generativer Ansätze muss erklärt werden können, wie das angeborene syntaktische Wissen aktiviert werden kann, da die Einheiten der Objektsprache nicht erkennbar ausgezeichnet sind. Anders formuliert: Wenn man annimmt, dass dem Kind Wissen über spezifisch sprachliche Repräsentationen angeboren ist, muss dieses Wissen in der Sprache eindeutig aktivierbar sein, da man ja explizit davon ausgeht, dass dieses sprachliche Wissen nicht durch Abstraktion über den Input konstruiert wird. Pinker löst das Linking-Problem, indem er annimmt, dass die Semantik eines Verbs die nötigen Kategorien liefert, um sein syntaktisches Verhalten eindeutig vorherzusagen: „Sentences conform to the demands of words in them because of general principles" (Pinker 1989, S. 4).

Pinker folgt hier lexikalistischen Theorien wie LFG (Lexical Functional Grammar) und HPSG (Head-Driven Phrase Structure Grammar), die ein intelligentes, hochstrukturiertes Lexikon annehmen (siehe auch Webelhuth, in diesem Band). Damit die Subkategorisierungseigenschaften eines Verbs ihre steuernde Kraft ausüben können, muss sowohl die Semantik des Verbs als auch der Zusammenhang zwischen Semantik und der Argumentstruktur erworben worden sein. Innerhalb generativer Ansätze geht man davon aus, dass ein entsprechendes Linking systematisch ist: Levin (1993) identifiziert 48 Gruppen mit 192 Unterkategorien für die Verben des Englischen auf Basis ihrer Semantik, sowie 79 Kategorien auf Basis ihrer Teilnahme an syntaktischen Alternierungen (Levin 1993, siehe auch Levin/ Rappaport Hovav 1995). Pinker (1989, 1994) stellte für den Spracherwerb die Hypothese auf, dass Linking-Regeln angeboren sein könnten. Das Kind müsste nur die semantischen Kasusrollen aus der Interaktion mit der Umwelt abstrahieren und könnte dann eine erfahrungsbasierte Kategorie wie 
„Agens“ mit der syntaktischen Realisierung „Subjekt“ verknüpfen. Nach dieser Hypothese bilden die semantisch zentralen Verben den Einstieg in den Syntaxerwerb, besonders der Argumentstruktur. In Anlehnung an die Arbeiten von Beth Levin und Malka Rappaport-Hovav (1995) wurde der englische Verbwortschatz in semantische Klassen eingeteilt, die jeweils mit syntaktischen Eigenschaften korrelieren, indem sie kraft ihrer Semantik die Syntax projizieren. Mit dem Konzept der semantischen Feineinteilung sollte insbesondere das schwer zu erklärende Phänomen der syntaktischen Alternierungen systematisch erfasst werden: Wieso alternieren einige Verben, obwohl semantisch eng verwandte Verben eine der Varianten nicht erlauben? Im Spracherwerb manifestiert sich dieses Problem durch Übergeneralisierungen (z.B. I disappeared the ball ,Ich verschwinde den Ball' für die Kausativ-Alternierung). In diesem Modell stehen Syntax und Semantik in einer direkten Abhängigkeitsbeziehung: Eine systematische Verknüpfung beider Ebenen erfolgt über das Linking von semantischen zu syntaktischen Rollen. Wenn diese Beziehung als eine systematische gedacht wird, ist es möglich, dass dem Kind das Wissen über eine Repräsentationsebene den Einstieg in die jeweils andere Struktur-Ebene erleichtert wird. Dies ist in den so genannten Theorien des Bootstrapping operationalisiert worden. Dieser aus der Informatik stammende Terminus bezeichnet den Prozess, mit dem ein Steuerungssystem einen komplexeren Prozess startet (Lindner/Hohenberger 2009, S. 230). Übertragen auf den Spracherwerb nahm man an, dass Wissen über einen Aspekt des Systems quasi als Starthilfe Lernprozesse auf einer anderen Ebene anstoßen kann. So wurde postuliert, dass die prosodische Gliederung systematische Hinweise auf die Grenzen syntaktischer Einheiten liefert, oder aber dass die Semantik den Einstieg in die syntaktische Repräsentation von Argumentstrukturen ermöglicht (siehe Höhle 2009 zur Diskussion verschiedener Formen des Bootstrapping). Im semantischen Bootstrapping sagten Gropen et al. (1991) für den Erwerb eine durch UG-Prinzipien gesteuerte Abfolge von „broad to narrow range rules“ vorher. Sie unterscheiden dabei zwischen „breiten“ Linking-Regeln, die als notwendige Bedingungen das syntaktische Verhalten semantischer Grobklassen wie Bewegungsverben steuern, und „engen“, stärker lexikalisierteren Linking-Regeln, die als hinreichende Bedingungen das syntaktische Verhalten bezogen auf feine semantische Unterschiede spezifizieren, z.B. bestimmte Arten der Bewegung. Mit Hilfe dieser Feinklassifizierung der Linking-Regeln sollte ein fehlerfreier Erwerb der Argumentstruktur möglich sein. Die Erwerbsaufgabe besteht in diesem Ansatz im Lernen der spezifischen Verbsemantik, die dann zur Anwendung der jeweils korrekten Linking-Regeln führen würde. Jedoch zeigten Analysen des Spracherwerbs englischer Kinder die postulierten Präferenzen für Verben mit kanonischer Argumentstruktur nicht, stattdessen finden sich früh Verben mit schwachem semantischen Gehalt (Bowerman 1990). 


\subsection{Argumentstruktur als Hilfe zum Erlernen der Verbsemantik}

Wenn eine enge Beziehung zwischen Syntax und Semantik besteht, sollte das Verhältnis auch umgekehrt nutzbar gemacht werden können. Im syntaktischen Bootstrapping geht man davon aus, dass das Verfolgen der syntaktischen Strukturen, in denen ein Verb benutzt wird, dem Kind hilft, die Verbbedeutung einzugrenzen bzw. zwischen verschiedenen Verbbedeutungen zu unterscheiden (Naigles 2002). Landau/Gleitmann (1985) fanden, dass selbst blinde Kinder die Bedeutung von Verben wie look und see aus ihrem Auftreten in bestimmten syntaktischen Kontexten abstrahieren können, obwohl ihnen die Wahrnehmungsdomäne nicht zugänglich ist.

Sowohl semantisches als auch syntaktisches Bootstrapping können nur dann funktionieren, wenn die Beziehungen zwischen Syntax und Bedeutung a priori und universal eindeutig spezifiziert sind:

The first proviso to the semantic usefulness of syntactic analysis for learning purposes is that the semantic/syntactic relations have to be materially the same across languages. Otherwise, depending on the exposure language, different children would have to perform completely different syntactic analyses to derive aspects of the meaning. (Gleitman 1990, S. 35)

Genau dies macht beide Ansätze inkompatibel (vgl. Bowerman/Brown 2008, S. 7-13), denn im semantischen Bootstrapping wäre die genaue Repräsentation der Semantik die Voraussetzung für den Erwerb der Syntax, während im syntaktischen Bootstrapping die Semantik erst gelernt werden muss, und die syntaktische Repräsentation als durch die Universalgrammatik angeboren angenommen wird. Zudem ließen sich die postulierten Syntax-Semantik-Verbindungen in sprachvergleichenden Untersuchungen nicht bestätigen (siehe die Artikel im Sammelband Bowerman/Brown (Hg.) 2008). In der Nachfolge dieser Diskussion ist Bootstrapping mehr und mehr zu einer Metapher für implizite Lernprozesse geworden (siehe Höhle 2009). Jedoch kann man die sehr differenzierten Argumente in dieser Diskussion nur dann wirklich evaluieren, wenn man sie in ihrer definitorischen Schärfe betrachtet: Pinker sah seine Theorie der Linking-Regeln als einen Mechanismus mit notwendigen und hinreichenden Bedingungen, nicht als technisierende Metapher für allgemeine Generalisierungsprozesse. Als Alternative zu den Bootstrapping-Mechanismen haben sich in der Folge konstruktivistische und gebrauchsbasierte Ansätze entwickelt, die den Erwerb der Argumentstruktur als graduellen Erwerbsprozess sehen.

\subsection{Lexikon und Grammatik in konstruktivistischen Ansätzen}

Konstruktivistischen Ansätzen liegt eine andere Hypothese von Erwerbsverläufen zu Grunde. Da sie nicht annehmen, dass spezifisches semantisches oder syntaktisches Wissen angeboren ist, muss beides aus dem Sprachge- 
brauch im weiteren Sinne, d.h. einschließlich seiner situativen und diskursiven Gebundenheit, abstrahiert werden. Ausgangspunkt sind Lernmechanismen, die auch in anderen Domänen attestiert sind. Der Vorteil ist, dass man keinen Dualismus zwischen angeborenen Erwerbsmechanismen und Lernmechanismen für den nicht angeborenen Rest annehmen muss (Tomasello 1998a; Behrens 2009b). Diese Grundannahmen implizieren anfangs graduelle, kontextspezifische Lernprozesse, da gerade nicht angenommen wird, dass mit einem Item die Kategorie als Ganzes aktiviert wird. Im Laufe der Entwicklung wird über die Wahrnehmung konkreter und relationaler Analogien das sprachliche Wissen stets abstrakter und generalisierter. Dies führt dazu, dass in der späteren Entwicklung auch sehr schnelle Lernprozesse möglich sind, sofern die zu lernende Struktur, etwa das Passiv, genügend Unterstützung durch bereits erworbene Konstruktionen, etwa die Perfekt-Konstruktionen, hat (Abbot-Smith/Behrens 2006).

Sprachtheoretisch beruht dieser Ansatz auf Grundannahmen der Kognitiven Linguistik (Langacker 1987), nach der alle sprachlichen Strukturen Form-Funktionspaare, also symbolische Einheiten sind. Dieses Konzept ist in den gebrauchsbasierten Versionen der Konstruktionsgrammatik umgesetzt. Das Verhältnis von Lexikon und Grammatik wird daher nicht als modular gesehen, wie im „words and rules“-Ansatz, sondern als Kontinuum, bei dem konkrete Morpheme und Wörter auf der einen Seite stehen, grammatisch abstrakte (lexikalisch variable) auf der anderen (Tabelle 1):

\begin{tabular}{|l|l|l|}
\hline \multicolumn{1}{|c|}{ Konstruktion } & \multicolumn{1}{|c|}{ Form/Beispiel } & \multicolumn{1}{|c|}{$\begin{array}{c}\text { Bedeutung (sofern } \\
\text { nicht transparent) }\end{array}$} \\
\hline Morphem & zer-, vor-, anti-, -lich, -te & \\
\hline Wort & Avocado, Anaconda, und & \\
\hline komplexes Wort & Fenerteufel, sit-in & Den Vogel abschießen \\
\hline Idiom (fixiert) & $\begin{array}{l}\text { In die X kriegen } \\
\text { (X= Haare, Wolle) }\end{array}$ & sich streiten \\
\hline Idiom (partiell fixiert) & je X desto Y & verbindet Variablen \\
\hline $\begin{array}{l}\text { Covariational conditional } \\
\text { construction }\end{array}$ & $\begin{array}{l}\text { Form: Subj V Dat-Obj } \\
\text { Akk-Obj / Er backte ibr } \\
\text { einen Kuchen }\end{array}$ & $\begin{array}{l}\text { Transfer (beabsichtigt } \\
\text { oder aktuell) }\end{array}$ \\
\hline ditransitive Konstruktion & $\begin{array}{l}\text { Form: Subj werden (PP) } \\
\text { Partizip Perfekt / Das } \\
\text { Gürteltier wurde von einem } \\
\text { Auto überfahren }\end{array}$ & $\begin{array}{l}\text { Diskursfunktion: einen } \\
\text { Patiens (undergoer) zum } \\
\text { Topic oder einen Agens } \\
\text { zum nicht-Topik machen }\end{array}$ \\
\hline Passiv
\end{tabular}

Tab. 1: Beispiele für Konstruktionen verschiedener Größe und Komplexität (nach Goldberg 2003, S. 220) 
Dass grammatische Strukturen Bedeutungsträger sein können, zeigt sich eindrücklich am Phänomen der coercion (Zwang, Nötigung), wenn die Bedeutung der Konstruktion die Lesart des Lexems verändert und die „eigentliche" Argumentstruktur des Verbs verletzt (z.B. I dreamt bim a cappucino ,ich (er)träumte ihm einen Cappuccino' oder be sneezed the napkin off the table ,er nieste die Serviette vom Tisch ${ }^{6}$. Trotz der Verletzung der Argumentstruktur des Verbs sind diese Sätze nicht nur eindeutig und relativ mühelos interpretierbar, sondern auch produktiv (er hustete das Bällchen aus der Röhre). Die präferierten Lesarten sind veränderbar und frequenzabhängig: Analysen des tatsächlichen Sprachgebrauchs zeigen in vielen Domänen statistische Schieflagen (,statistical skewing“", ,statistical biases"), insofern als nicht alle Zellen eines Paradigmas gleichmäßig besetzt sein müssen, und insofern als statistische Gebrauchspräferenzen die Lesart einer prinzipiell polysemen Struktur beeinflussen können. So wird im Englischen die Konstruktion „Verb - Objekt - lokative PP“ mit der Bedeutung der verursachten Bewegung im konkreten und metaphorischen Sinn assoziiert (he got he ball over the fence), während die Doppel-Objekt-Konstruktion mit der Bedeutung „konkreter" oder „metaphorischer Transfer“ assoziiert ist (be got him a cake) (Goldberg 2006).

Für den Spracherwerb bedeuten diese Grundannahmen, dass man ihn holistisch konzeptualisieren kann: Kinder erwerben nicht die Wörter mit ihren Subkategorisierungseigenschaften, die dann bestimmen, an welchen syntaktischen Strukturen ein Wort partizipieren kann, sondern sie erwerben das Lexikon durch und in den Konstruktionen, in denen sie auftauchen. In konstruktivistischen Theorien werden Syntax-Semantik-Beziehungen auf Basis einer Reihe von allgemeinen Generalisierungsmechanismen abstrahiert:

constructionist theories propose that mapping generalizations are learned from the input, with learning constrained by pragmatics, categorization principles, attentional biases, and other domain-general factors. (Boyd/Gottschalk/Goldberg 2009, S. 65)

Studien zu intransitiven, transitiven, ditransitiven und anderen Argumentstrukturrelationen haben gezeigt, dass der Erwerb einer Struktur zunächst an einzelne oder wenige Lexeme gebunden ist, dass es weder in der Distribution natürlicher Erwerbsdaten noch in Experimenten weite Generalisierungen über den attestierten Gebrauch hinaus gibt (Tomasello 2003). Im Laufe der Entwicklung lockert sich die Bindung von Strukturen an bestimmte lexikalische Einheiten oder Cluster. Der Erwerb von Syntax und Lexikon ist im Sinne der gebrauchsbasierten Konstruktionsgrammatik also als eine Abstraktion über Gebrauchsereignisse zu sehen.

Dies impliziert auch eine Loslösung vom klassischen Konzept des Valenzrahmens, da dieser keine Aussagen über deren konkrete syntaktische Realisierung macht (Behrens 2009a). Allen/Skarabela/Hughes (2008) zeigen, dass die konkrete Argumentrealisierung sprachtypologische Variation zeigt und auch durch diskurspragmatische Faktoren bedingt ist. 


\section{Konstruktionen als Einstieg in den Syntaxerwerb}

In der gebrauchsbasierten Variante der Konstruktionsgrammatik geht man davon aus, dass sprachliche Kategorien im Spracherwerbsprozess auf Basis des sprachlichen Inputs und der Interaktion geformt und gelernt werden. Dies schließt nicht aus, dass angeborenes oder in der vorsprachlichen Phase erworbenes Wissen eine Rolle spielen kann, jedoch ist das Forschungsziel, die Prozesse der sprachlichen (Über)Formung von Konzepten zu ermitteln. ${ }^{2}$

Bezogen auf das Sprachsystem heißt dies, dass sowohl die semantischen und formalen Eigenschaften der lexikalischen Einheiten als auch die Struktur des Sprachsystems aus dem Sprachgebrauch abstrahiert werden müssen. Die Konstruktionsgrammatik bringt dabei auch „top down“-Prozesse holistischen Lernens ins Spiel, wenn das Kind mit größeren Einheiten (,,chunks") beginnt und diese erst im Verlauf der Sprachentwicklung analysiert. Holistische Lernprozesse sind auch früher schon postuliert worden, galten aber als nicht-produktiv in einem Lernprozess, der im Wesentlichen als „bottom up"-Strategie von einfachen zu komplexen Strukturen gesehen wurde. Auf den ersten Blick scheint die Tatsache, dass die meisten Kinder mit Einwortäußerungen beginnen und peu à peu Zwei- und Dreiwortstrukturen erlernen, den holistischen Strategien zu widersprechen. Jedoch sind die Einwortäußerungen und die darauffolgenden frühen Zweiwortäußerungen syntaktisch nicht komplex, sondern - funktional gesehen - strukturell ungebundene Aneinanderreihungen von Wörtern (Papa weg, Mama Socke). Distributionsanalysen der frühen Wortkombinationen zeigen, dass diese Kombinationen nur eine geringe Variabilität aufweisen, also wenig produktiv sind. Dies zeigt sich daran, dass eine sehr geringe Anzahl von Wortkombinationen den weitaus größten Teil der Tokens einer bestimmten Struktur ausmacht. Pine/Lieven/Rowland (1998) ermittelten, dass die fünf häufigsten Subjekt-Verb-Schemata (z.B. go $+X$ oder Mommy $+X)$ durchschnittlich 75,7\% der Kombinationen bei zwölf zwei- bis dreijährigen englischen Kindern stellten.

\subsection{Lexikalisch-spezifische Erwerbsverläufe}

Michael Tomasellos Studie zum Verb-Erwerb seiner Tochter „T“ (First verbs, 1992) bildete den Ausgangspunkt für die modernen konstruktivistischen Ansätze. Er führte eine detaillierte Tagebuchstudie zum syntaktischen

\footnotetext{
Zur Kategorienbildung vgl. insbesondere die Forschung zur sprachlichen Relativität: Hier zeigen Experimente, dass Kinder im Alter von 9-14 Monaten an unterschiedliche räumliche Kategorisierungen habituiert werden können, jedoch Kinder mit 2-3 Jahren die Kategorisierung ihrer Muttersprache übernommen haben und andere nicht mehr wahrnehmen (McDonough/Choi/Mandler 2003). Analog zu Befunden in der Lautdiskriminierung werden diese Resultate so gewertet, dass Kinder eine sehr flexible Lernfähigkeit haben, die unterschiedliche Kategorisierungen sprachlicher und nicht-sprachlicher Stimuli erlaubt.
} 
Gebrauch von Verben und stellte fest, dass sich die Syntagmen der verschiedenen Verben, die sie im zweiten Lebensjahr produzierte, kaum überschneiden, sondern jedes Verb einen eigenen Strukturaufbau zu durchlaufen schien und eine Insel der Entwicklung bildete. Tomasello prägte für diesen lexikalisch-spezifischen Entwicklungsverlauf die Metapher der Verb-Inseln. Spätere Studien zeigten, dass auch Pronomina eine solche Insel-Funktion ausüben können (Pine/Lieven/Rowland 1998). Die „verb island“-Нypothese präjudiziert also nicht, dass den Verben per se eine privilegierte Rolle im Strukturaufbau zukommt, und sollte daher allgemeiner als lexem-spezifisches („lexically-specific“) Lernen gefasst werden, ${ }^{3}$ um zu betonen, dass das sich entwickelnde sprachliche Wissen zunächst um das konkrete Item selbst, nicht um seine Kategorie aufgebaut wird. So besteht der Valenzrahmen des Verbs këussen zunächst aus den Aktanten kisser/kissee statt der abstrakteren Relationen Agens/Patiens oder Subjekt/Objekt. Evidenz für diese Annahme ist die Distribution in natürlichsprachlichen Daten: Wenn Verben mit der gleichen Argumentstruktur mit unterschiedlichen Argumenten realisiert werden, gilt dies als Indiz dafür, dass die Verben nicht miteinander vernetzt sind. Der Inselstatus ist auch experimentell überprüfbar, indem man testet, inwieweit und unter welchen Bedingungen Kinder Strukturen mit ,nonce“-Wörtern, also solchen, die sie nie vorher gehört haben, auf neue Situationen übertragen: In Trainingsstudien zeigt sich, dass Kinder anfänglich nicht in der Lage sind, über den dargebotenen Subkategorisierungsrahmen hinauszugehen, sondern Strukturen so reproduzieren, wie sie sie gehört haben (siehe Tomasello 2003 für eine Übersichtsdarstellung). Wenn transitive Verben intransitiv präsentiert werden (Look! He's meeking!), findet anfangs keine Generalisierung von intransitiv zu transitiv statt (What's he doing? He's meeking. Aber nicht: He's meeking the cow). Derselbe Effekt findet sich im umgekehrten Paradigma (Training mit transitiven Strukturen, Zielstruktur intransitiv, Tomasello/Brooks 1998). In so genannten „weird word order"-Experimenten werden neue Verben zu transitiven Aktionen in ungrammatischer Wortstellung präsentiert (im Englischen SOV oder VSO). Vierjährige korrigieren die Wortstellung zuverlässig auf SVO, Zweijährige halten sich überwiegend an die jeweils gehörte Wortstellung (Akthar 1999). Auch Priming-Experimente zeigen verb-spezifische Entwicklungen. Priming bezeichnet den Effekt, dass das Hören des Wortes oder einer Struktur die Wiederauftretenswahrscheinlichkeit im Sinne einer Bahnung erhöht, weil das Wort oder die Struktur bereits aktiviert ist. Wenn Vierjährige eine erhöhte Input-Frequenz an Passiv-Sätzen erhalten, produzieren sie danach auch mehr Passivsätze, aber nur solche mit dem gleichen lexikalischen Material.

Das Konzept des Lexikons wird auch auf noch unanalysierte „chunks“ erweitert, die als Ganzes eine bestimmte Funktion enkodieren, z.B. Fragestrukturen wie where's the X oder wo's $\operatorname{der} X$. 
Bei Sechsjährigen hingegen findet sich auch strukturelles Priming: Hier erhöht sich die Zahl der Passivsätze auch mit anderem lexikalischen Material. Dies gilt als Evidenz für strukturelles Priming (Savage et al. 2003).

Die Forschung zur Produktivität und zum Grad der Generalisierung zeigt, dass Kinder in den frühen Phasen weit weniger generativ und kreativ sind als bislang postuliert. Der Eindruck, dass Kinder Sprache effizient und weitgehend fehlerfrei erlernen, lässt sich durchaus durch konservative Lernmechanismen erklären: Kinder experimentieren gerade nicht, indem sie mit bekanntem lexikalischen Material regelgeleitet neue Strukturen produzieren, sondern sie halten sich im Wesentlichen an die Modelle, die sie im Input vorfinden, um die kommunikativen Anforderungen zu bewältigen. Sie scheitern zunächst jedoch, wenn sie bekannte Strukturen auf neue Wörter übertragen müssen. Tomasello (2000) zeigte, dass selbst die syntaktisch vermeintlich einfachen Strukturen transitiver Sätze erst mit neun Jahren in allen Aspekten gemeistert werden.

\subsection{Der Erwerb der Verbzweit-Wortstellung}

Wie lassen sich Phänomene im Strukturaufbau durch die Konstruktionsgrammatik erfassen, die in ihrer Regelmäßigkeit Lexem-übergreifend sind? Als ein solches Phänomen kann die deutsche (und niederländische) Satzklammer gelten: eine syntaktische Eigenschaft, die unabhängig vom Lexikon zu sehen ist, denn prinzipiell unterliegen alle Verben diesem Strukturprinzip. Im frühen Sprachgebrauch deutscher und niederländischer Kinder gibt es jedoch Unterschiede zwischen Kopula- und Modalverben, die fast ausschließlich in finiter Form vorliegen (Jordens 1990), und den Vollverben, die je nach Flexion in V1/V2 oder verbfinaler Position auftreten. Während der Erwerb der Satzklammer den meisten Zweitsprachlernern Probleme bereitet, finden sich im Erstspracherwerb sich normal entwickelnder Kinder wenig Fehler bezogen auf den Zusammenhang von Finitheit und V1/V2Stellung (siehe Tracy in diesem Band). Doch vollzieht sich in den frühen Phasen des Spracherwerbs ein Wandel von einem System, das weitgehend durch infinite Vollverben gekennzeichnet ist (aufmachen; Katze holen etc.), zu einem finiten System.

Aus gebrauchsbasierter und konstruktionsgrammatischer Perspektive ergeben sich also die folgenden Forschungsfragen: Wie ist die Evidenz im Input für die Satzklammer? Zu unterscheiden wären Sätze mit einfachem, finiten Verb und solche mit zweiteiligem Verb, in denen die Satzklammer sichtbar wird. Weiterhin ist die Nebensatzstellung zu beachten. In welchen Entwicklungsschritten vollzieht sich der Wortstellungserwerb?

Das V2-Phänomen ist ein „Klassiker“ der Spracherwerbsforschung zum Deutschen und Niederländischen. Im Fokus steht die Korrelation zwischen Finitheitsmarker und Verbstellung. Die Erwerbsreihenfolge ist in der nor- 
malen Sprachentwicklung so, dass anfangs vor allem nicht-finite Verbformen auftreten, zunächst vorwiegend in Isolation, später in Kombination mit meist vorangestellten Objekten, Subjekten oder anderen Elementen (Negation, Adverbien) (siehe Beleg (1)). Finite Formen sind selten, hier treten - im Niederländischen - anfänglich vor allem Kopula, Modalverben und statische Verben auf (Jordens 1990) sowie eher formelhafte und imitierte Konstruktionen (2), wobei es verschiedene Thesen zur funktionalen Unterscheidung finiter und nicht-finiter Formen gibt (vgl. Blom 2008). Wenige Monate nach den ersten Infinitiven tauchen erste komplexe Verbformen auf, sowohl getrennte Partikelverbkombinationen, modale Infinitive und Perfektkonstruktionen mit Auxiliar und Partizip Präsens (3). ${ }^{4}$
a. Leo 2;0.1 schließen
b. Leo
$2 ; 0.20$
Tisch decken
a. Leo
$2 ; 0.3$
passt durch
b. Leo
$2 ; 0.5$
guckt müde
a. Leo
$2 ; 2.7$
b. Leo
$2 ; 2.15$
kann man Zug bauen
da is $(t)$ durchgefahren

Bemerkenswert ist, dass es nach anfänglichen Schwankungen kaum Fehler in der Wortstellung gibt. In der „Principles \& Parameters"-Version der generativen Grammatik war der V2-Effekt ein potenzieller Parameter, unter der Annahme, dass bestimmte morphologische Merkmale des Verbs (z.B. Tempus, Aspekt, Modalität) die Bewegung des finiten Verbs aus der VerbletztPosition lizenzieren (siehe Lasser 2002 und Blom 2008 zu den diskutierten Varianten). Jedoch gibt es bislang keinen Konsens über die genaue Spezifikation eines solchen Parameters.

Auf den ersten Blick scheint sich die Erwerbsreihenfolge auch nicht aus dem Input erklären zu lassen, denn reine Infinitive oder Partizipien sind in der Erwachsenensprache selten und nur unter bestimmten Bedingungen möglich (Stillgestanden! Alle aufwachen!) (vgl. Lasser 1997). Infinitive und Partizipien sind aber nur dann selten, wenn man sie als eigenständige Struktur mit strikt spezifizierter morphologischer Repräsentation sieht, nicht als Teil komplexer Verbformen. Eine Auszählung des morphologisch kodierten Teilkorpus (500.000 Wörter) aus dem Leo-Korpus ergab, dass rund 35\% aller im Input vorkommenden Verben mehrteilig sind (z.B. modale Infinitive und

$4 \quad$ Die hier und im Folgenden zitierten Kindersprachdaten entstammen der Leipziger „,dense database“ zum Erwerb des Deutschen. Die Sprachentwicklung des monolingualen Jungen Leo wurde vom 2. bis zum 5. Lebensjahr mit einer erhöhten Aufnahmefrequenz von 5 Stunden/Woche im 3. Lebensjahr und 5 Stunden/Woche während einer Woche pro Monat im 4. und 5. Lebensjahr dokumentiert. Die Gesamtgröße des Korpus umfasst ca. 500.000 Wörter von Leo, 1.3 Millionen Wörter im Input (siehe Behrens 2006 für weitere Details). Die Altersangaben sind in JJ.MM.TT angegeben. Das Korpus ist Teil des öffentlich zugänglichen CHILDES-Archivs (MacWhinney 2000). 
Perfektkonstruktionen). Da Verben eine frequente Kategorie sind und diese Formen an salienter finaler Position stehen, ist wahrnehmungspsychologisch durchaus zu begründen, warum diese Formen als erstes aufgegriffen werden: Sie sind inhaltstragend, perzeptuell salient und frequent (Behrens 1993). In einer Reihe von Computersimulationen eines konstruktivistischen Lernmodells (MOSAIC $=$,Model of Syntax Acquisition In Children“) simulierten Freudenthal/Pine/Gobet (2006, 2009) und Freudenthal et al. (2007) den Verbstellungserwerb im Deutschen, Englischen, Niederländischen, Französischen und Spanischen, indem sie nur die Wahrnehmungsfaktoren Position und Länge des nötigen Arbeitsspeichers modifizierten. Theoretische Basis sind die „less is more“- (Newport 1990) und die „starting small“"Hypothese (Elman 1993), nach denen der begrenzte Arbeitsspeicher von Kleinkindern ihnen hilft, in das Sprachsystem einzusteigen, weil es ihre Aufmerksamkeit natürlicherweise auf die Relation zwischen eng zusammenstehenden Elementen lenkt, während sie zunächst scheitern, Relationen zwischen diskontinuierlichen Elementen zu erkennen (Newport/Aslin 2004). Das MOSAIC-Modell arbeitet ohne vorspezifiziertes sprachliches Wissen auf Basis von Input-Daten aus dem CHILDES-Archiv (MacWhinney 2000). Unter der Annahme, dass das zuletzt Gehörte in der Wahrnehmung am aktivsten ist, verarbeitet das Modell den Input von rechts nach links und produziert als Output Äußerungen zunehmender Länge. Dieser Output kann dann mit kindersprachlichen Erwerbsdaten gleicher Äußerungslänge verglichen werden. Es zeigt sich, dass das Modell Strukturen produzierte, die mit den natürlichen Erwerbssequenzen in diesen Sprachen hochgradig übereinstimmen. Die sprachspezifische Passung konnte noch erhöht werden, indem man einen zweiten Aufmerksamkeitsfokus auf den ebenfalls salienten Äußerungsbeginn modellierte, um den unterschiedlichen Wortstellungsmustern der analysierten Sprachen Rechnung zu tragen. Auch dies entspricht natürlichen Erwerbsverläufen, weil Kinder lernen müssen, wo welche Information enkodiert ist, und welche Positionen in ihrer Sprache besonders relevant sind.

Diese Computersimulationen konkreter Erwerbsverläufe in verschiedenen Sprachen zeigen also, dass wenige Faktoren (Modifikation der Aufmerksamkeitsspanne und des Aufmerksamkeitsfokus) ausreichen, um den Erwerb der Wortstellung vorherzusagen, auch ohne angeborenes Wissen annehmen zu müssen. Dieser Ansatz, der den Spracherwerb mit nur wenigen, psychologisch plausiblen Grundannahmen erklärt, wird kontrastiert mit generativen Ansätzen, die den Verbzweit-Erwerb als inkrementelle Aktivierung angeborener Parameter ansehen. Hier wären nicht die semantischen Eigenschaften des Verbs entscheidend für den Syntaxaufbau wie in der Argumentstruktur, sondern die morphologischen Merkmale des Verbs, die die Bewegung aus der verbfinalen Position lizenzieren (siehe Lasser 1997 und 2002 für eine Zusammenschau der vorgeschlagenen Repräsentationen). 
Lässt sich der Aufbau der Wortstellungsmuster auch in natürlichen Erwerbsdaten beobachten? Die Analyse des großen Leo-Korpus (siehe Fußnote 4) scheint dies zu bestätigen. Behrens (2010) analysierte den Erwerb der Wortstellung aus der Perspektive des Aufbaus des Mittelfelds. Da frühe Kindersprache durch kurze Äußerungen charakterisiert ist, ist die attestierte korrekte Wortstellung nicht überraschend, denn bei Äußerungslängen von 2-3 Wörtern ist die Wahrscheinlichkeit von Wortstellungsfehlern gering, insbesondere dann, wenn man wie im gebrauchsbasierten Paradigma davon ausgeht, dass sich Kinder zunächst konservativ an attestierte Strukturen halten. Von daher scheint es erhellender, Äußerungen zu analysieren, bei denen das Mittelfeld besetzt ist, so dass es im Prinzip eine höhere Wahrscheinlichkeit von Wortstellungsfehlern gibt. Die Auswertungen zeigen, dass das Mittelfeld nur sehr langsam aufgebaut wird: Zunächst stehen Verb und Partikel oder Auxiliar - Partizip/Infinitiv nebeneinander (4), bevor Klitika oder kurze Einzelwörter eingeschoben werden (5):
a. Leo
$1 ; 11.27$
komm mit
b. Leo
$2 ; 00.29$
nicht pack weg

Größere Konstituenten tauchen in Kombination mit komplexen Verben erst drei Monate nach dem Auftreten in Isolation auf (6). Da das Deutsche eine relativ große Variation in der Wortstellung erlaubt, findet man kaum explizite Wortstellungsfehler (4b), sondern eher Strukturen, die pragmatisch unangemessen sind (7).

$$
\text { Leo 2;02.5 }
$$

ein Zug fäbrt ab

Leo $\quad 2 ; 02.29$

\section{Tilli und Bärbel haben getroffen}

,wir haben Tilli und Bärbel (beim Spaziergang) getroffen'

Auf den ersten Blick scheinen die Daten also den schnellen und fehlerfreien Erwerb der deutschen Wortstellung zu bestätigen. Allerdings kommt die Fehlerfreiheit auch dadurch zustande, dass die betreffenden Strukturen in den ersten Monaten des Auftretens extrem selten sind: Im Alter von 2;3 etwa enthalten circa 3.000 der knapp 11.000 aufgezeichneten Äußerungen ein Verb, davon sind 348 komplexe Verbformen. Daneben sind 1.900 komplexe, minimal aus zwei Wörtern bestehende Nominalphrasen attestiert. Aber nur in 53 Äußerungen, also 5 Promille der Gesamtäußerungen, kommen mehrteilige Verben und komplexe NPs zusammen vor. Im Alter von 2;5 beträgt der Anteil solcher komplexen, aber im Deutschen durchaus üblichen Strukturen 2\%. Zieht man die Kürze der meisten Äußerungen in Betracht, bieten sich kaum Möglichkeiten, überhaupt Fehler zu machen. Insofern sind die Daten mit Theorien kompatibel, die von einer langsamen Genera- 
lisierung ausgehen, zumal der Input hinreichende Evidenz für dieses komplexe, aber hochfrequente Phänomen liefert. Im Sinne des „competition model“" (MacWhinney 2004) hätten wir es hier also mit hohen Verarbeitungskosten (,high cue cost“), aber auch hoher Verfügbarkeit („cue availability") zu tun, zumal der hohe Verarbeitungsaufwand nur dann vorliegt, wenn man das Gesamtsystem betrachtet. Sobald perzeptive Filter wie etwa im MOSAIC-Modell angenommen werden, reduziert sich die Komplexität der jeweils zu verarbeitenden Information erheblich.

Für künftige Forschungen an spontanen Korpora wäre es aufschlussreich, nicht nur nach attestierten Strukturen und ihrer Produktivität zu schauen, sondern auch das Fehlende zu ermitteln. Welche Aspekte des Systems sind noch nicht erworben, obwohl einzelne oder alle Aspekte dieser Strukturen bereits in einigen Kontexten gemeistert werden? Dieses Verfahren lässt sich exemplarisch am Aufbau lexemspezifischen Wissens demonstrieren.

\subsection{Aufbau von Verb-Grammatiken}

Das Verb reparieren ist ein einfaches transitives Verb mit konkreter Bedeutung und von einiger Relevanz im Leben eines Kleinkindes. Die 421 Belege im Leo-Korpus konzentrieren sich dann auch auf die frühen Phasen, als Leo zu reparierende Dinge benennt oder aber Andere zur Reparatur auffordert. Betrachtet man die Argumentrealisierung der einzelnen Wortformen, stellt man folgende Unterschiede fest: Die mit Abstand häufigste Form ist der reine Infinitiv (Abbildung 1), in der Regel ohne weitere Argumente gebraucht, ab 2;3 jedoch auch in Kombination mit Objekt-NP (8a-d) und - seltener - Subjekt-NP (8e) jedoch nie mit Subjekt und Objekt. Die Wortstellung ist dabei variabel $(8 \mathrm{a}-\mathrm{b})$. Die Funktion ist vor allem die einer Bitte oder einer Aufforderung.

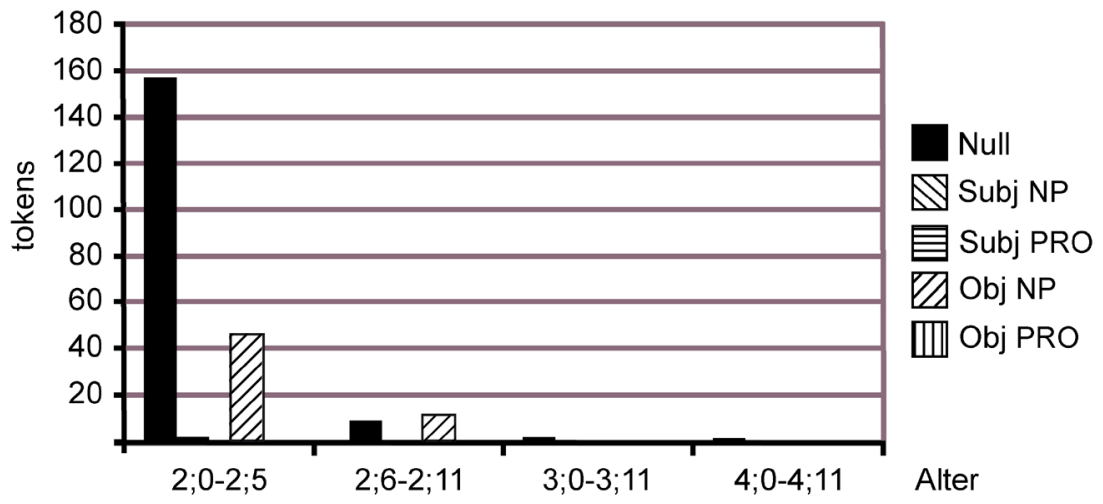

Abb. 1: Argumentrealisierung bei reparieren als Infinitiv 
(8)
a. Leo $2 ; 3.8$
Brïcke reparieren
b. Leo $2 ; 3.8$ reparieren die Brücke
c. Leo $2 ; 3.6$
$I+C+$ E reparieren
d. Leo 2;3.6
Bus reparieren
e. Leo 2;3.7
Mama reparieren

Als modaler Infinitiv (Abbildung 2) tritt reparieren auch mit Aufforderungsoder Verpflichtungscharakter auf (9), wobei zunächst nur der Agens enkodiert wird.
a. Leo
$2 ; 2.14$
ich muss auch reparieren
b. Leo
$2 ; 2.25$
beute xxx, Papa Arb(eit), Papa Papa muss reparieren

Erst mit knapp 2;5 finden sich erste Belege mit Besetzung von Subjekt und Objekt, wobei das Subjekt typischerweise mit einem Eigennamen, das Objekt pronominal realisiert ist (10a, b). Der in den elterlichen Tagebuchnotizen attestierte Beleg (10c) über den Wiederaufbau der Dresdner Frauenkirche stellt den Höhepunkt an Komplexität der Belege dar, wobei auch hier die Argumentrealisierung von reparieren selbst wenig komplex ist.
a. Leo 2;4.24 Ernie soll den reparieren
b. Leo $2 ; 4.23$
muss Ernie den reparieren
c. Leo $2 ; 9.24$ wenn man sieht, dass eine Franenkirche kaputt ist, muss
man die sofort reparieren (Tagebuchnotiz)

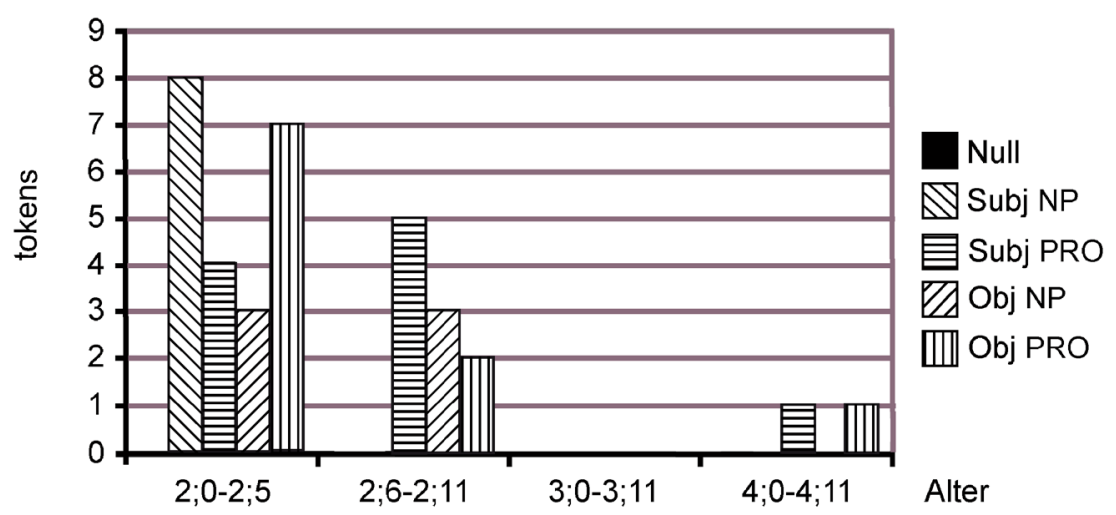

Abb. 2: Argumentrealisierung bei reparieren als Modalinfinitiv

Präsensformen treten ab 2;1.27 auf (Abbildung 3), auch sie zunächst in reduzierter Argumentstruktur (11), bis ab 2;3.20 volle Argumentstrukturen auftreten (12). ${ }^{5}$ Im Präsens kommentiert Leo vornehmlich die aktuelle Aktivität.

\footnotetext{
In den Abbildungen sind nur die jeweiligen Realisierungen von Subjekt und Objekt erfasst, nicht deren Kombinatorik, was zu einer zu großen Komplexität in der Darstellung geführt hätte.
} 


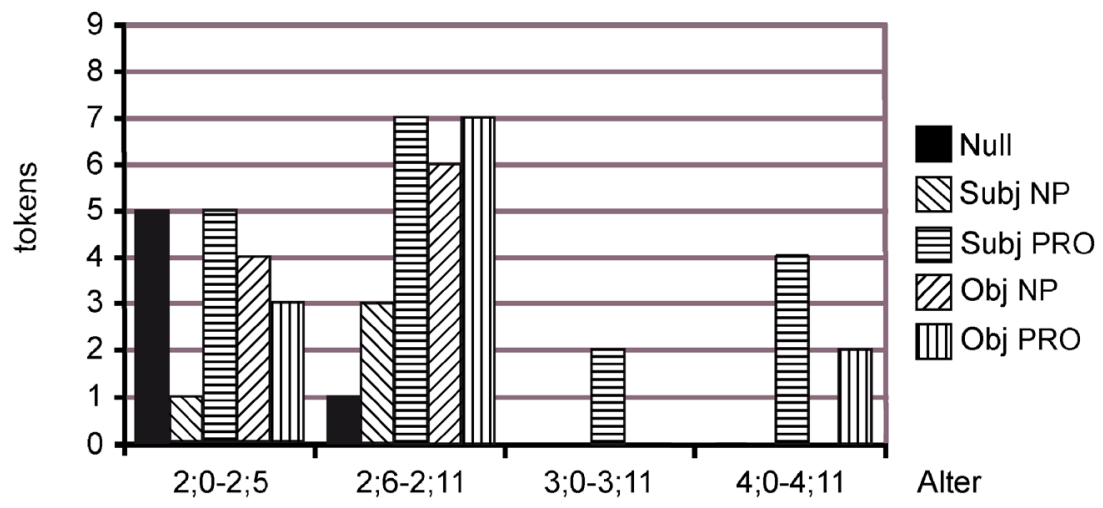

Abb. 3: Argumentrealisierung bei reparieren im Präsens
a. Leo
$2 ; 1.27$
reparierst
b. Leo
2.2 .16
Papa repariert
c. Leo
$2 ; 3.19$
den Tunnel repariert
$\begin{array}{ll}\text { a. Leo } & 2 ; 3.20 \\ \text { b. Leo } & 2 ; 3.29 \\ \text { c. Leo } & 2 ; 6.24\end{array}$
ich repariere die Lampe! (Tagebuchnotiz)
der re(pa)riert ein Auto
und die reparieren ein Lichtschalter, die reparieren ein neuen Lichtschalter

Wie schon bei den Daten zum Erwerb der Verbzweitstellung zeigt sich auch hier ein sehr langsamer Strukturaufbau und eine geringe Variabilität der verwendeten Strukturen, sobald man Lexeme oder Strukturen im Detail analysiert. Analysen dieser Art sind mit traditionellen Korpora (30 oder 60 Minuten Aufnahme alle drei oder vier Wochen) kaum durchführbar, da einzelne Items nicht oft genug attestiert sind. So entsteht fast zwangsläufig der Eindruck großer Produktivität, da fast jeder der oft weit auseinander liegenden Belege neue Strukturen und Kookkurenzpartner zeigt. Wenn die Hypothese vom graduellen Strukturaufbau zutrifft, sollte also die Stereotypizität der Äußerungen in größeren Korpora stärker hervortreten, obwohl die Wahrscheinlichkeit der Variabilität in großen Datenmengen größer ist (zum Zusammenhang zwischen Sampling-Rate und der Wahrscheinlichkeit des Auftretens einer Struktur siehe Rowland/Fletcher/Freudenthal 2008).

Eine Methode zur Messung des Grads der syntaktischen Produktivität ist das so genannte „Traceback“" Hier werden die Daten eines Tages dahingehend analysiert, wie groß der Anteil der vorher noch nicht im Korpus attestierten Äußerungen ist, und in welchem Grade und in welcher Art die neuen Äußerungen von den bereits attestierten Äußerungen abweichen (Lieven et al. 2003). Bei einem englischen Kind waren $63 \%$ der mit 2;1.11 attestierten Äußerungen in genau dieser Form schon in einem der Transkripte der 
sechs vorhergehenden Wochen belegt. Von den verbleibenden 109 neuen Äußerungen wiesen über 70\% nur eine minimale Modifikation zu vorher attestierten Äußerungen auf (Einfügen, Weglassen oder Ersetzen eines Wortes oder einer Wortgruppe). Die Übereinstimmung mit vorher Gesagtem wird noch größer, wenn man die Funktion einbezieht (Dąbrowska/Lieven 2005). Die Validität dieses Fortgehens lässt sich testen, indem man die Modifikationsschritte des vorherigen Sprachgebrauchs nimmt, um daraus Vorhersagen über die nächsten Entwicklungsschritte abzuleiten und zu testen (,traceforward“, Bannard/Lieven 2009).

\section{Wie umfassend erklärt Konstruktionsgrammatik den Spracherwerb?}

In dem theoretisch polarisierten Feld der Spracherwerbsforschung ist die am häufigsten gehörte Frage in Diskussionen die, ob Konstruktionsgrammatik tatsächlich Grammatikerwerb erklären könne, der sich in der generativen Sichtweise weitgehend unabhängig von Sprachgebrauch und Bedeutung manifestiert. Aus der Sichtweise gebrauchsbasierter Modelle stellt sich diese Frage nicht, denn grammatische Strukturen werden aus dem Sprachgebrauch abstrahiert, bestehen nicht a priori in irgendeiner mentalen Repräsentation, die nur noch aktiviert bzw. reifen muss. Im Gegenzug stellt sich die Frage, was denn das generative Modell noch erklärt. Im Modell der Prinzipienund Parametertheorie wurden sehr spezifische Vorhersagen zur Universalität sprachlicher Strukturen und zum Spielraum sprachspezifisch zu setzender Parameter gemacht, die aber letztendlich nicht bestätigt werden konnten. Der Minimalismus macht sehr viel sparsamere Vorhersagen zu universalen Strukturen, so dass der Raum dessen, was sprachspezifisch gelernt werden muss, eher größer als kleiner geworden ist. Wie oben angedeutet, ist die praktisch und theoretisch unbefriedigende Konsequenz, dass man so nicht nur zwei Erwerbsmechanismen annehmen muss (einen für die Aktivierung angeborener Strukturen, einen klassischen Lernmechanismus für den Rest), sondern dem Kind ja auch irgendein Wissen angeboren sein muss in Bezug darauf, welchen Mechanismus es denn auf welche Struktur anzuwenden hat. Bezogen auf den Wortstellungserwerb im Deutschen lässt sich sagen, dass das Phänomen der Satzklammer hochgradig regelmäßig und hochgradig präsent ist, insofern als es sich in jedem deutschen Satz mit Verb manifestiert. In der Terminologie des „,competition model“ hätte es also eine hohe „cue strength“ (MacWhinney 2004), gemessen an der Verfügbarkeit (Frequenz) und Verlässlichkeit (Eindeutigkeit). Dass Kinder dieses Phänomen recht zielsicher erwerben, ist also nicht verwunderlich. Dass es ein langwieriger und in seinen Einzelheiten ein eher lokaler als globaler Prozess ist, im Lichte der UG schon. Die oben gezeigten Befunde zum Einstieg in ein- 
fache Satzstrukturen gelten auch für den Erwerb komplexer Sätze: Auch hier ist die Wortstellung in Abhängigkeit des Komplementierers nur ein Aspekt des Erwerbs von Nebensatzkonstruktionen. Genauere Analysen zeigen, dass es einen graduellen Erwerb der Funktion und der an dieser Struktur beteiligten Lexeme gibt: Bei Verben des Meinens und Denkens etwa fungieren erste Strukturen wie I think im Englischen oder ich glaub im Deutschen eher als Satzadverbiale denn als Verben, die einen Komplementsatz subkategorisieren (vgl. Diessel 2004; Brandt/Diessel/Tomasello 2008). Konstruktivistische und konstruktionsgrammatische Ansätze zum Spracherwerb erlauben es, die Funktion der Strukturen in konkreten Diskurssituationen einzubeziehen. Grundlage für den Erwerb sprachlicher Strukturen ist die Äußerung in ihrer kontextgebundenen Manifestation.

\section{Literatur}

Abbot-Smith, Kirsten/Behrens, Heike (2006): How known construction influence the acquisition of other constructions: the German periphrastic passive and future constructions. In: Cognitive Science 30, S. 995-1026.

Akhtar, Nameera (1999): Acquiring basic word order: evidence for data-driven learning of syntactic structure. In: Journal of Child Language 26, S. 339-356.

Allen, Shanley/Skarabela, Barbora/Hughes, Mary (2008): Using corpora to examine discourse effects in syntax. In: Behrens (Hg.), S. 99-137.

Bannard, Colin/Lieven, Elena (2009): Repetition and reuse in child language learning. In: Corrigan, Roberta et al. (Hg.): Formulaic language. Bd. II: Acquisition, loss, psychological reality, functional explanations. Amsterdam/Philadelphia, S. 297-321.

Behrens, Heike (1993): Temporal reference in German child language: form and function of early verb use. Diss. Univ. Amsterdam.

Behrens, Heike (2006): The input-output relationship in first language acquisition. In: Language and Cognitive Processes 21, S. 2-24.

Behrens, Heike (Hg.) (2008): Corpora in language acquisition research: Finding structure in data. (= Trends in Language Acquisition Research 6). Amsterdam/Philadelphia.

Behrens, Heike (2009a): Konstruktionen im Spracherwerb. In: Zeitschrift für Germanistische Linguistik 20, S. 427-444.

Behrens, Heike (2009b). Usage-based and emergentist approaches to language acquisition. In: Linguistics 47, S. 383-411.

Behrens, Heike (2010): Acquiring the middle field: word-order in German. Ms. Basel.

Blom, Elma (2008): The acquisition of finiteness. (= Studies in Generative Grammar 94). Berlin/New York.

Bowerman, Melissa (1990): Mapping thematic roles onto syntactic functions: are children helped by linking rules? In: Linguistics 28, S. 1253-1290. 


\section{AUTHOR'S COPY | AUTORENEXEMPLAR}

Bowerman, Melissa/Brown, Penelope (2008): Introduction. In: Bowerman/Brown (Hg.), S. 1-26.

Bowerman, Melissa/Brown, Penelope (Hg.) (2008): Crosslinguistic perspectives on argument structure: implications for language acquisition. Mahwah.

Boyd, Jeremy K./Gottschalk, Erin A./Goldberg, Adele E. (2009): Linking rule acquisition in novel phrasal constructions. In: Language Learning 59, S. 64-89.

Brandt, Silke/Diessel, Holger/Tomasello, Michael (2008): The acquisition of German relative clauses: a case study. In: Journal of Child Language 35, S. 325-348.

Brown, Penelope (1998): Children's first verbs in Tzeltal: evidence from the early verb category. In: Linguistics 36, S. 713-753.

Dąbrowska, Ewa/Lieven, Elena (2005): Towards a lexically specific grammar of children's question constructions. In: Cognitive Linguistics 16, S. 437-474.

Diessel, Holger (2004): The acquisition of complex sentences. (= Cambridge Studies in Linguistics 105). Cambridge u.a.

Elman, Jeffrey L. (1993): Learning and development in neural networks: the importance of starting small. In: Cognition 48, S. 71-99.

Freudenthal, Daniel/Pine, Julian M./Gobet, Fernand (2006): Unifying cross-linguistic and within-language patterns of finiteness marking in MOSAIC. In: Sun, Ray/ Miyake, Naomi (Hg.): Proceedings of the 28th Annual Meeting of the Cognitive Science Society. Mahwah, S. 232-236.

Freudenthal, Daniel et al. (2007): Modelling the developmental patterning of finiteness marking in English, Dutch, German and Spanish using MOSAIC. In: Cognitive Science 31, S. 311-341.

Freudenthal, Daniel/Pine, Julian M./Gobet, Fernand (2009): Simulating the referential properties of Dutch, German and English Root Infinitives in MOSAIC. In: Language Learning and Development 5, S. 1-29

Gentner, Dedre (1978): On relational meaning: the acquisition of verb meaning. In: Child Development 49, S. 988-998.

Gleitman, Lila R. (1990): The structural sources of verb meaning. In: Language Acquisition 1, S. 3-55.

Goldberg, Adele E. (2003): Constructions: a new theoretical approach to language. In: Trends in Cognitive Sciences 7, S. 219-224.

Goldberg, Adele E. (2006): Constructions at work: the nature of generalization in language. Oxford.

Gropen, Jess et al. (1991): Affectedness and direct objects: the role of lexical semantics in the acquisition of verb argument structure. In: Cognition 41, S. 153-195.

Hohenberger, Annette/Peltzer-Karpf, Annemarie (2009): Language learning from the perspective of nonlinear dynamic systems. In: Linguistics 47, S. 481-511.

Höhle, Barbara (2009): Bootstrapping mechanisms in first language acquisition. In: Linguistics 47, S. 359-382. 


\section{AUTHOR'S COPY | AUTORENEXEMPLAR}

Grammatik und Lexikon im Spracherwerb: Konstruktionsprozesse

Jordens, Peter (1990): The acquisition of verb placement in Dutch and German. In: Linguistics 28, S. 1407-1448.

Landau, Barbara/Gleitman, Lila R. (1985): Language and experience: evidence from the blind child. Cambridge, MA.

Langacker, Ronald W. (1987): Foundations of cognitive grammar. Bd. 1: Theoretical prerequisites. Stanford.

Lasser, Ingeborg (1997): Finiteness in adult and child German. (= MPI Series in Psycholinguistics 8). Nijmegen.

Lasser, Ingeborg (2002): The roots of root infinitives: remarks on infinitival main clauses in adult and child language. In: Linguistics 40, S. 767-796.

Levin, Beth (1993): English verb classes and alternations: a preliminary analysis. Chicago.

Levin, Beth/Rappaport-Hovav, Malka (1995): Unaccusativity: at the the syntax-lexical semantics interface. (= Linguistic Inquiry Monographs 26). Cambridge, MA.

Lieven, Elena et al. (2003): Early syntactic creativity: a usage-based approach. In: Journal of Child Language 30, S. 333-370.

Lindner, Katrin/Hohenberger, Annette (2009): Introduction: concepts of development, learning, and acquisition. In: Linguistics 47, S. 211-239.

MacWhinney, Brian (2000): The CHILDES Project: tools for analyzing talk. 2 Bde. 3. Aufl. Mahwah.

MacWhinney, Brian (2004): New directions in the competition model. In: Slobin, Dan I./ Tomasello, Michael (Hg.): Beyond nature-nurture: essays in honor of Elizabeth Bates. Mahwah, S. 81-110.

McDonough, Laraine/Choi, Soonja/Mandler, Jean M. (2003): Understanding spatial relations: flexible infants, lexical adults.In: Cognitive Psychology 46, S. 229-259.

Naigles, Letitia R. (2002): Form is easy, meaning is hard: resolving a paradox in early child language. In: Cognition 86, S. 157-199.

Newport, Elissa L. (1990): Maturational constraints on language learning. In: Cognitive Science 14, S. 11-28.

Newport, Elissa L./Aslin, Richard N. (2004): Learning at a distance: I. Statistical learning of non-adjacent dependencies. In: Cognitive Psychology 48, S. 127-162.

Pine, Julian M./Lieven, Elena V.M./Rowland, Caroline F. (1998): Comparing different models of the English verb category. In: Linguistics 36, S. 807-830.

Pinker, Steven (1987): The bootstrapping problem in language acquisition. In: MacWhinney, Brian (Hg.): Mechanisms of language acquisition. Hillsdale, S. 399-441.

Pinker, Steven (1989): Learnability and cognition: the acquisition of argument structure. Cambridge, MA.

Pinker, Steven (1994): How could a child use verb syntax to learn verb semantics? In: Lingua 92, S. 377-410. 


\section{AUTHOR'S COPY | AUTORENEXEMPLAR}

396 Heike Behrens

Rowland, Caroline F./Fletcher, Sarah L./Freudenthal, Daniel (2008): How big is big enough? Assessing the reliability of data from naturalistic samples. In: Behrens (Hg.), S. 1-24.

Savage, Ceri et al. (2003): Testing the abstractness of children's linguistic representation: lexical and structural priming of syntactic constructions in young children. In: Developmental Science 6, S. 557-567.

Tomasello, Michael (1992): First verbs: a case study of early grammatical development. Cambridge.

Tomasello, Michael (1998a): Introduction: a cognitive-functional perspective on language structure. In: Tomasello, Michael (Hg.): The new psychology of language: cognitive and functional approaches to language structure. Mahwah, S. vii-xxiii.

Tomasello, Michael (1998b): The return of constructions. Review Essay on: Goldberg, A. (1995). ,Constructions: a construction grammar approach to argument structure'. In: Journal of Child Language 25, S. 443-484.

Tomasello, Michael (2000): Do young children have adult syntactic competence? In: Cognition 74, S. 209-253.

Tomasello, Michael (2003): Constructing a language: a usage-based account of language acquisition. Cambridge, MA.

Tomasello, Michael/Brooks, Patricia J. (1998): Young children's earliest transitive and intransitive constructions. In: Cognitive Linguistics 9, S. 379-395. 